\title{
Sedação consciente endovenosa com midazolam no tratamento odontológico de pessoas com deficiência
}

\author{
Intravenously conscious sedation with midazolam to odontological \\ treatment in disable persons \\ Sedación intravenosa consciente con midazolam para el tratamiento \\ odontológico en personas discapacitadas \\ Sandra Maria H. Coelho Ávila de AGUIAR ${ }^{1}$ \\ Alessandra Marcondes ARANEGA ${ }^{\mathbf{1}}$ \\ Taís Elisabete Crivellaro de MENEZES ${ }^{2}$ \\ Fátima Hassan Baz LAURETTO ${ }^{3}$ \\ Antonio Donizeti SOARES ${ }^{4}$ \\ Stélios FIKARIS ${ }^{4}$ \\ ${ }^{1}$ Docentes da Faculdade de Odontologia de Araçatuba - UNESP \\ ${ }^{2}$ Doutoranda em Odontopediatria, Faculdade de Odontologia de Araçatuba - UNESP \\ ${ }^{3}$ Cirurgiã-Dentista do Centro de Assistência Odontológica a Pessoa com Deficiência-UNESP \\ ${ }^{4}$ Médicos do Centro de Assistência Odontológica a Pessoa com Deficiência - UNESP
}

\section{Resumo}

O objetivo deste estudo foi observar os resultados dos procedimentos dentários realizados em pessoas com deficiência sob sedação consciente com midazolam, por via intravenosa, atendidas no Centro de Assistência Odontológica para Pacientes com Deficiências, da Faculdade de Odontologia de Araçatuba - UNESP. Foram observados 460 procedimentos, independentemente do sexo, idade ou deficiência dos pacientes, que não permitiam a realização do tratamento odontológico rotineiro. Os resultados foram classificados como sucesso (quando realizados sem problemas, mesmo que fosse necessária restrição física suave, ou havia algum problema contornável, mas os procedimentos planejados puderam ser realizados) e insucesso (quando não foi possível realizar o tratamento planejado). Também foram registrados o uso diário de medicamentos pelos pacientes e prováveis causas de resultados negativos. A pressão arterial, a frequência cardíaca, a saturação de oxigênio e a temperatura corporal foram monitoradas antes, durante e após o procedimento. As doses de midazolam foram administradas entre $0,5 \mathrm{e} 1 \mathrm{mg} / \mathrm{kg}$, de acordo com o peso corporal e a reação do paciente. $\mathrm{Na}$ maioria dos casos, foi possível realizar o tratamento planejado sem problemas para os pacientes ou para a equipe odontológica e seus sinais vitais permaneceram dentro dos valores normais. Portanto, conclui-se que a sedação consciente com midazolam, por via intravenosa, pode ser considerada uma alternativa efetiva para tratamento odontológico de pessoas com deficiência não colaboradoras.

Descritores: Pessoas com deficiência; Sedação Consciente; Assistência Odontológica; Midazolam; Pacientes.

\begin{abstract}
The objective of this study was to observe the results of dental procedures performed in disable persons under conscious sedation with midazolam, intravenously, assisted at the Dental Care Center for Patients with Special Needs, School of Dentistry of Araçatuba - UNESP. 460 procedures were observed, regardless of gender, age or disability of the patients, which did not allow their dental treatment realization. The results were classified as success (when carried out without problems, even it was necessary restraint gentle help, or there was some manageable problem, but the planned procedures could be performed) and failure (when it was not possible to perform the planned treatment). It was also recorded, the use of medicines by patients and the probable causes of failure results. Blood pressure, heart rate, oxygen saturation and body temperature were monitored before, during and after the procedure. Doses of midazolam were administered between 0.5 and $1 \mathrm{mg} / \mathrm{kg}$, according to the patient's body weight and behavioral reaction. In most cases it was possible to perform the planned treatment without problems to the patients or to the dental staff and their vital signs remained within normal values. The conscious sedation with midazolam, intravenously, can be considered an effective alternative for dental treatment to disable persons not employees.
\end{abstract}

Descriptors: Disable Persons; Conscious Sedation; Dental Care; Midazolam; Patients

\section{Resumen}

El objetivo de este estudio fue observar los resultados de los procedimientos dentales realizados en personas com deficiencia bajo sedación consciente con midazolam, por vía intravenosa, atendidas en el Centro de Assistencia Odontologica para Pacientes con Necesidades Especiales, Facultad de Odontología de Araçatuba - UNESP. Se observaron 460 procedimientos, independientemente del sexo, la edad o la discapacidad de los pacientes, que no permitieron la realización de su tratamiento dental. Los resultados se clasificaron como exitosos (cuando se fueron realizados sin problemas, incluso quando tu ez necesaria una restricción física suave, o hubo algún problema manejable, se pudieron realizar los procedimientos planeados) y el fracaso (cuando no fue posible realizar el tratamiento planeado). También se registró el uso de medicamentos por parte de los pacientes y las posibles causas de los resultados negativos. La presión arterial, la frecuencia cardíaca, la saturación de oxígeno y la temperatura corporal se controlaron antes, durante y después del procedimiento. Las dosis de midazolam se administraron entre $0,5 \mathrm{y} 1 \mathrm{mg} / \mathrm{kg}$, de acuerdo con el peso corporal del paciente y la reacción conductual. En la mayoría de los casos, fue posible realizar el tratamiento planeado sin problemas para los pacientes o para el equipo médico y sus signos vitales se mantuvieron dentro de los valores normales. Por lo tanto, se concluye que la sedación intravenosa con midazolam intravenoso puede considerarse una alternativa efectiva para el tratamiento dental de personas con deficiencia que no colaboran

Descriptores: Personas con Discapacidad; Sedación Consciente; Atención Odontológica; Midazolam; Pacientes

\section{INTRODUÇÃO}

Sedação é o procedimento que leva ao aprofundamento do nível de consciência do paciente, através da utilização de medicações sedativas com o objetivo primordial de permitir o tratamento odontológico. Essas medicações promovem a redução controlada do nível de consciência e discretamente na percepção da dor, mantendo os parâmetros hemodinâmicos estáveis e respiração espontânea ${ }^{1}$. De acordo com a intensidade em que será empregada, poderá ser consciente ou profunda ${ }^{2,3}$.
Sedação consciente é a expressão empregada para descrever estados controlados de diminuição do nível de consciência, em geral mantendo-se reflexos protetores, como ventilação respiratória, deglutição e até mesmo a tosse. Enfim, o paciente mantém respostas apropriadas a estímulos físicos e comandos verbais. Ela pode ser empregada para a realização de procedimentos radiológicos (ex. ressonância magnética), clínicos (ex. a troca de um cateter), odontológicos ou cirúrgicos para diminuir o 
desconforto e ansiedade do paciente, facilitando a execução e proporcionando melhor qualidade e segurança dos procedimentos $^{2-4}$.

Já a sedação profunda inclui a perda de reflexos protetores das vias aéreas e possível comprometimento da função miocárdica. Nestas condições os pacientes podem sofrer diminuição da oxigenação sanguínea ou aspirar conteúdos gástricos ${ }^{2,3}$.

Em tratamentos odontológicos, grande parte dos pacientes apresentam medo e ansiedade ${ }^{3,5-7}$, sentimentos que podem ser exacerbados por procedimentos que geram dor $^{3,7,8}$. Embora muitas técnicas de condicionamento de comportamento possam ser empregadas, existe uma parcela de pacientes ansiosos ou apreensivos, com deficiências físicas e/ou mentais, que necessitam de métodos adjuvantes para viabilizar o tratamento odontológico ${ }^{1,3,7}$.

$\mathrm{Na}$ maioria dos casos, a sedação consciente é o procedimento de escolha e, para este fim, diversos tipos de drogas podem ser utilizadas, tais como o óxido nitroso ${ }^{6,9,10}$ o hidrato de cloral ${ }^{11,12}$. Mas, atualmente, os benzodiazepínicos constituem o grupo farmacológico mais utilizados por apresentarem uma boa efetividade $\mathrm{e}$ segurança $\mathrm{a}^{3,5,6,13}$.

$\mathrm{O}$ midazolam apresenta as características farmacológicas que o tornaram como medicamento de escolha para a sedação na clínica, tais como: início de ação rápida, meia vida curta e duração da sedação adequada. Seus efeitos ansiolíticos, hipnóticos e amnésicos estão entre os objetivos principais a serem alcançados com sua utilização. Além disso, a diversidade de vias de administração como: oral, retal, intramuscular, endovenosa e intranasal oferecem alternativas que se adaptam à grande maioria dos pacientes. ${ }^{3,14}$

A utilização correta e segura de medicamentos depressores do sistema nervoso central sempre requer conhecimento da sua farmacologia e treinamento em suporte básico e avançado em ressuscitação cardiopulmonar por parte do cirurgião-dentista e sua equipe de trabalho ${ }^{3}$.

Em decorrência da escassa literatura sobre sedação com midazolam em pacientes com deficiência e, devido aos bons resultados obtidos, o presente estudo teve como objetivo analisar a efetividade da sedação consciente com midazolam, via endovenosa, em pacientes com deficiência, para realização de tratamentos odontológicos.

\section{MATERIAL E MÉTODO}

Inicialmente este projeto foi encaminhado ao Comitê de Ética em Pesquisa Humana, da Faculdade de Odontologia do Campus de Araçatuba - UNESP, sendo aprovado através do processo FOA 2007-01342.

Para a realização deste trabalho foram acompanhados 460 pacientes submetidos a procedimentos de sedação com midazolam (SM), por via endovenosa (EV), selecionados aleatoriamente, entre os anos de 20082012, independentemente do gênero, idade ou tipo de deficiência dos mesmos, e realizados no Centro de Assistência Odontológica à Pessoa com Deficiência (CAOE), Unidade Auxiliar de Estrutura Complexa, da Faculdade de Odontologia do campus de Araçatuba, da Universidade Estadual Paulista "Júlio de Mesquita Filho"UNESP, na cidade de Araçatuba, S.P, Brasil.

Os pacientes selecionados haviam demonstrado comportamento negativo às técnicas de controle comportamental ou apresentavam espasticidade muscular (pacientes com Paralisia Cerebral, em sua grande maioria), colocando em risco sua integridade física e/ou da equipe odontológica para a realização dos procedimentos odontológicos necessários.

Cada procedimento foi avaliado através de uma ficha "padrão" (Figura 1) desenvolvida com base em levantamento bibliográfico sobre os efeitos do uso de midazolam EV, em procedimentos de sedação ${ }^{13,15,16}$.

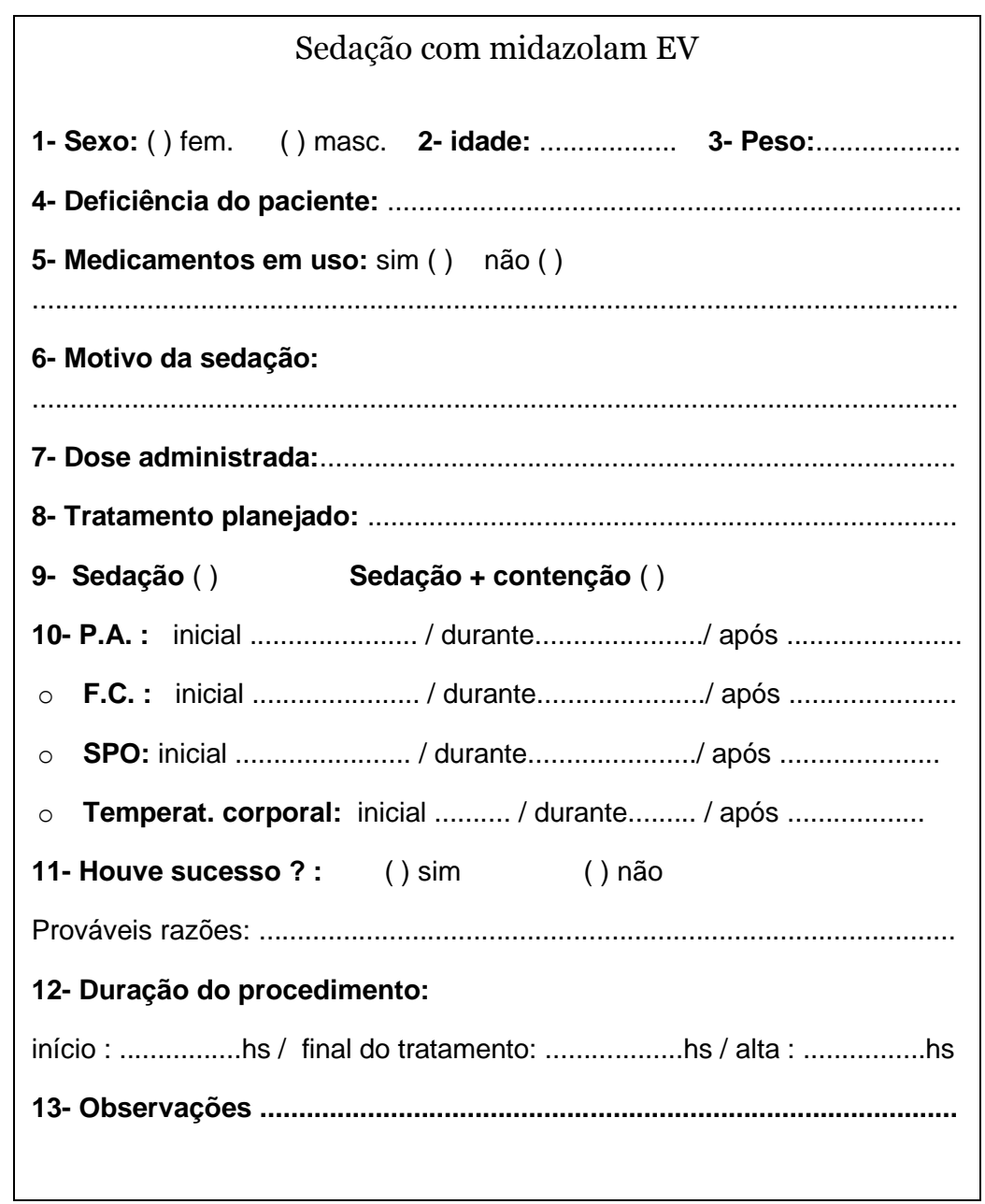

Figura 1: Ficha padrão utilizada para avaliação dos procedimentos de sedação.

O procedimento de sedação consciente com midazolam foi considerado bem sucedido quando o tratamento odontológico planejado pôde ser realizado até o final, ainda que fosse necessária contenção adicional ou houvesse algum efeito adverso contornável e, as situações classificadas como insucesso foram aquelas em que não foi possível a realização do tratamento planejado. Nesses casos, o paciente foi encaminhado para tratamento sob anestesia geral (AG).

Todos os pacientes foram acompanhados desde o período pré-operatório até o momento de sua alta, por uma equipe composta por médico, enfermeiras, cirurgiãodentista e auxiliares técnicos odontológicos e de enfermagem.

Os pacientes chegavam em jejum de 8 horas e, após serem pesados, seus prontuários médico-odontológico analisados, sinais vitais monitorados e, constatada uma boa condição geral de saúde pela equipe médica-odontológica, eram submetidos à sedação consciente com midazolam utilizando-se uma dose inicial de $0,5 \mathrm{mg} / \mathrm{kg}$ de midazolam, podendo ser aumentada se necessário, com doses adicionais, para até no máximo $1,0 \mathrm{mg} / \mathrm{kg}$, de acordo com a resposta, principalmente em pacientes mais resistentes à droga ou devido a procedimentos odontológicos maiores que levam mais tempo de duração que o habitualmente realizado.

As doses utilizadas são geralmente mais elevadas nesses pacientes do que aquelas utilizadas em pacientes sem deficiência intelectual ${ }^{17-18}$. Em consonância com a literatura internacional nossos resultados também demonstraram a necessidade de doses mais elevadas dos 
sedativos para obtenção de um adequado nível de sedação ${ }^{19}$. A razão pela qual os pacientes com deficiência intelectual mais frequentemente necessitam de uma dose mais alta de midazolam do que outros pacientes não está clara, porém nossos pacientes frequentemente são usuários de outros medicamentos para controle de seus comportamento, às vezes em altas doses; geralmente esses são os mesmos que requerem doses maiores de midazolam para sedação; não se pode determinar até que ponto a interação medicamentosa do midazolam com essas outras drogas podem interferir na farmacocinética e farmacodinâmica $^{17}$. Independentemente de interação medicamentosa, o efeito sedativo do midazolam tem grande variabilidade individual ${ }^{9}$.

Após cerca de 2 minutos da infusão lenta do midazolam, e constatado o efeito da droga administrada, estando o paciente bem e sedado, o tratamento odontológico planejado era iniciado.

Todos os equipamentos e medicamentos necessários para atendimento de emergência, caso fosse preciso, estavam disponíveis, assim como profissionais devidamente preparados.

Os parâmetros hemodinâmicos e respiratórios eram monitorados no pré, trans e pós-operatórios, através de monitores cardíacos e oxímetros. Oxigenoterapia era instituída quando a saturação de oxigênio, medida pelo oxímetro digital, caia abaixo de $94 \%$ de saturação, com 5 litros de oxigênio, por minuto, mantido enquanto fosse necessário para a consecução do tratamento. Após o término do atendimento odontológico, caso o paciente tenha permanecido sedado após o procedimento, utilizavase o antagonista de benzodiazepínico, o flumazenil $\mathrm{EV}^{*}$ em dose preconizada pelo fabricante do medicamento. Destaca-se que o fármaco, registrado e fabricado por Cristália - Produtos Químicos Farmacêuticos Ltda, está indicado para anular, completa ou parcialmente, os efeitos sedativos centrais dos benzodiazepínicos. Em seguida o paciente era encaminhado à sala de recuperação, monitorado e assim até a total recuperação da consciência, reflexos e a não apresentação de sangramentos, liberando então para os familiares ou cuidadores para a alta e volta a sua residência.

Os dados obtidos foram tabulados em planilhas do programa Microsoft Excel® para conferência, confecção de tabelas e posterior análise dos resultados.

\section{RESULTADOS}

Foram acompanhados 460 procedimentos de sedação consciente com midazolam, via endovenosa, em pacientes que apresentavam entre 02 e 60 anos de idade, sendo 219 (47,6\% do total) do sexo feminino e $241(52,4 \%$ do total) do masculino.

Com relação ao tipo de deficiência apresentada pelos 460 pacientes selecionados observou-se que: $49 \%$ apresentavam distúrbio neuropsicomotor (DNPM); $35 \%$ possuíam paralisia cerebral (PC) e $16 \%$ apresentavam algum tipo de síndrome ou doença genética, sendo a Síndrome de Down, a maioria nestes casos.

Quanto ao motivo de encaminhamento para a sedação consciente com midazolam, pôde-se notar que 378 pacientes (82,2\% do total) apresentaram comportamento não-colaborador por uma ou mais vezes, resistindo às técnicas utilizadas de manejo de comportamento e 74 pacientes $(16,1 \%$ do total) foram encaminhados por apresentar espasticidade muscular, sendo que, destes, 71 $(15,4 \%$ do total) com paralisia cerebral. Quanto aos demais
(1,7\% do total), o motivo variou entre reações adversas como vômitos e sialorreia excessiva durante o tratamento. Foi realizado um total de 460 procedimentos odontológicos, envolvendo tratamentos de periodontia, cirurgia (exodontias, em sua grande maioria), dentística (restaurações diretas e selantes, em sua grande maioria) e endodontia.

Os atendimentos odontológicos apresentaram duração entre 15 e 60 minutos, sendo que, a duração média foi de 30 a 40 minutos (223 atendimentos - 48,5\%) e a dose média utilizada para a sedação consciente com midazolam $(\mathrm{EV})$ foi de $0,91 \mathrm{mg}$ por quilo de peso corporal.

Tabela 1. Índice de sucesso apresentado pelos diferentes grupos

\begin{tabular}{c|c|c|c}
\hline Grupo & $\begin{array}{c}\text { Sucesso } \\
(\mathbf{n} \div) /(\%)\end{array}$ & $\begin{array}{c}\text { Insucesso } \\
(\mathbf{n} \div) /(\%)\end{array}$ & $\begin{array}{c}\text { Totais } \\
\text { (parciais) }\end{array}$ \\
\hline D.N.P.M. & $218 / 92,5$ & $8 / 3,5$ & 226 \\
\hline P.C. & $151 / 98,1$ & $3 / 1,9$ & 154 \\
\hline Sindrômicos & $80 / 100$ & $0 / 0$ & 80 \\
\hline Total & $449 / 97,6$ & $11 / 2,4$ & 460
\end{tabular}

Em relação ao grupo em que se obteve sucesso, apenas 168 (36,5\% do total) necessitaram de contenção auxiliar ou apresentaram algum tipo de reação adversa contornável. Os pacientes em que não se obteve sucesso foram encaminhados para tratamento odontológico sob anestesia geral.

Quanto à necessidade de contenção auxiliar no total de procedimentos acompanhados, pôde-se notar que em 280 pacientes (60,9\% do total) não se fez necessário.

Quanto à necessidade de contenção auxiliar no total de procedimentos acompanhados, pôde-se notar que em 280 pacientes (60,9\% do total) não se fez necessário.

Tabela 2. Relação entre o uso de medicamentos e o índice de sucesso

\begin{tabular}{c|c|c}
\hline Uso de medicamentos & $\begin{array}{c}\text { C/ sucesso } \\
\left(\mathbf{n}^{\circ}\right) /(\%)\end{array}$ & $\begin{array}{c}\text { S/ sucesso } \\
\left(\mathbf{n}^{\circ}\right) /(\%)\end{array}$ \\
\hline sim & $293 / 63,7$ & $7 / 1,5$ \\
\hline não & $156 / 33,9$ & $4 / 0,9$ \\
\hline Total & $449 / 97,6$ & $11 / 2,4$ \\
\hline
\end{tabular}

Entre os pacientes que faziam uso de medicamentos e obtiveram sucesso, 106 (23\% do total) necessitaram de contenção auxiliar para finalizar o tratamento; e entre os que não faziam uso de medicamentos e obtiveram sucesso, 62 (13,5 \% do total) necessitaram de contenção auxiliar para finalizar o tratamento.

Tabela 3. Relação de efeitos adversos encontrados no total de procedimentos de sedação consciente com midazolam

\begin{tabular}{l|c|c}
\hline \multicolumn{1}{c|}{ Efeitos adversos } & $\begin{array}{c}\text { Episódios } \\
\text { (número) }\end{array}$ & $\begin{array}{c}\text { Porcentagem (em } \\
\text { relação ao total de } \\
\text { SCM) }\end{array}$ \\
\hline $\begin{array}{l}\text { Queda de saturação de } \\
\text { oxigênio }\end{array}$ & 17 & $3,7 \%$ \\
\hline Náuseas/vômito & 08 & $1,7 \%$ \\
\hline Soluços & 07 & $1,5 \%$ \\
\hline Tosse & 01 & $0,2 \%$ \\
\hline Secreção pulmonar & 04 & $0,9 \%$ \\
\hline Taquicardia (>100bpm) & 01 & $0,2 \%$ \\
\hline TOTAL & 38 & $8,2 \%$ \\
\hline
\end{tabular}

Em relação aos efeitos adversos apresentados na Tabela 3, em nenhum caso foi necessário atendimento de emergência. Alguns pacientes apresentaram mais que um efeito adverso. 
Tabela 4. Relação de médias dos sinais vitais acompanhados ao longo dos procedimentos.

\begin{tabular}{c|c|c|c}
\hline Sinal Vital & Antes & Durante & Após \\
\hline Pressão arterial $(\mathbf{m m H g})$ & $112 \times 70$ & $118 \times 76$ & $107 \times 71$ \\
\hline Frequência cardíaca $(\mathbf{b p m})$ & 95 & 118 & 86 \\
\hline Saturação de $\mathbf{O}_{2}(\%)$ & 96 & 92 & 98 \\
\hline Temperatura corporal $\left({ }^{\circ} \mathbf{C}\right)$ & 36,4 & 36,3 & 36,5 \\
\hline
\end{tabular}

\section{DISCUSSÃO}

A sedação com midazolam consciente se mostrou um procedimento seguro e eficaz. Podendo ser considerado uma alternativa a cirurgia sob anestesia geral, uma vez que não se tem que utilizar leitos hospitalares, que em geral são escassos e limitados para esse perfil de pacientes que requerem estruturas adequadas de infraestruturas e pessoal treinados para atendimentos a esses pacientes. Assim mesmo que se tenha necessidade de se repetir a sedação pela segunda ou terceira vez, para se concluir todo o plano de tratamento, mas com o tempo de recuperação da sedação em geral rápida e alta no mesmo dia, com apenas algumas horas dentro do nosso serviço ${ }^{15,16}$

Muitas drogas têm sido utilizadas para esse tipo de sedação, tais como o óxido nitroso ${ }^{19-23}$ e o hidrato de cloral $^{24,25}$. Mas, dentre elas, o grupo dos benzodiazepínicos vem se destacando para este fim. E, dentro deste grupo, o midazolam tem demonstrado maior eficácia e segurança ${ }^{16,18,19,26}$. Suas características farmacológicas, tais como: início de ação rápida, meia vida curta, duração da sedação adequada, bons efeitos ansiolíticos, hipnóticos e amnésicos, baixo custo e existência de medicamento antagonista específico (flumazenil) estão entre os objetivos principais para a escolha desta droga. Além da diversidade de vias de administração como: oral, retal, intramuscular, endovenosa e intranasal, oferecendo alternativas que se adaptam à grande maioria dos pacientes ${ }^{16,27}$.

A seleção de pacientes para sedação com midazolam sem dúvida é um fator que está diretamente relacionado ao índice de sucesso do procedimento. Pacientes submetidos anteriormente à entubação prolongada, portadores de apnéia do sono ou de cardiopatias congênitas devem ser cuidadosamente acompanhados, assim como alguns autores contra-indicam o uso de midazolam em pacientes com hipersensibilidade a benzodiazepínicos e mulheres no primeiro trimestre de gestação. Ainda recomendam uso cauteloso em idosos, hepatopatas, lactantes, portadores de miastenia gravis e asma ${ }^{3,20,28}$. Deve-se ressaltar que jamais deixamos de fazer sedação em algum paciente, por conta de alguma patologia concomitante.

Salienta-se a importância da realização de uma boa anamnese, como ocorreu com todos os pacientes avaliados neste estudo, através da qual pôde-se detectar candidatos de risco para essa prática e/ou que pudessem exigir maior atenção da equipe médica que acompanhava os procedimentos. A realização de exame físico rigoroso, incluindo sinais vitais, ausculta cardíaca e pulmonar e avaliação de vias aéreas era fundamental ${ }^{3,24,25}$. Como complemento, exames laboratoriais eram pedidos rotineiramente (hemograma completo, glicemia, creatinina e coagulograma).

Nenhum tipo de deficiência encontrada interferiu positiva ou negativamente de maneira significativa para o índice de sucesso do procedimento sedativo avaliado, demonstrando segurança quanto ao uso deste medicamento em pacientes com deficiências.

Com relação à seleção dos pacientes encaminhados para a realização de tratamentos odontológicos sob sedação, o motivo da grande maioria dos casos observados, ou seja, em 378 deles, correspondendo a 82,2\% do total, foi o comportamento não colaborador, não permitindo a realização de seus tratamentos odontológicos por uma ou mais vezes, resistindo às técnicas utilizadas no manejo de comportamento. Observou-se, também, que 74 (16,1\% do total), foram encaminhados para sedação por apresentarem espasticidade muscular, sendo que a maioria deles eram portadores de paralisia cerebral (71 casos, correspondendo a $15,4 \%$ do total). Quanto aos demais (1,7\% do total), o motivo do encaminhamento variou entre ânsias excessivas durante o tratamento; crianças menores impossibilitando o manejo de comportamento, ou tratamento necessário muito extenso. Mediante estes resultados encontrados observamos que nenhum destes pacientes foi encaminhado para sedação indevidamente, sem tentativas ou justificativas previamente analisadas, evitando-se ainda, que os mesmos fossem submetidos a uma possível anestesia geral para realização do tratamento odontológico planejado.

Com relação ao gênero e a idade dos pacientes, não foram observadas interferências significantes durante $o$ procedimento de sedação consciente com midazolam (EV), que fossem dignas de registro neste estudo.

O índice de sucesso de 97,6\% (449 procedimentos) encontrado no presente estudo, associado ao fato de em apenas 36,5\% (168 casos) ter sido necessária contenção auxiliar ou ter ocorrido algum tipo de reação adversa contornável veio reforçar a segurança do uso do midazolam, desde que de maneira segura, para realização de sedação consciente para viabilização de tratamentos odontológicos em portadores de deficiência. Os 11 pacientes $(2,4 \%$ do total) em que não se obteve sucesso foram encaminhados para tratamento odontológico sob anestesia geral, permitindo que esse procedimento, de maior risco, fosse realizado apenas em pacientes que realmente não permitiram alternativas para realização de seu tratamento.

O fato de que em $60,9 \%$ (280 procedimentos) dos casos não ter sido necessária contenção auxiliar, sugere-se que as dosagens utilizadas e consideradas seguras ao paciente foram efetivas e suficientes para permitir a realização dos tratamentos odontológicos. E, mesmo nos casos onde se fez necessária a contenção, foi possível a realização do tratamento, pois se obteve maior facilidade no controle do paciente.

A respeito de interações medicamentosas, Ramos et al. ${ }^{16}$ relatam alguns medicamentos que merecem atenção, como: carbamazepina, fenitoína, rifampicina, roxitromicina, eritromicina, fluconazol, verapamil, entre outros. É importante salientar que $65,2 \%$ dos pacientes deste estudo faziam uso regular de medicamentos, inclusive alguns dos medicamentos acima citados, bem como drogas de efeito antagonista ao Midazolam. Apesar disso, cabe ressaltar que o índice de sucesso encontrado entre o grupo de pacientes que faziam uso de medicamentos rotineiros foi de $63,7 \%$ do total, muito significativo em relação ao baixo índice de insucesso (apenas 1,5\%), neste mesmo grupo de pacientes.

Dentre os $34,8 \%$ que não faziam uso de nenhum medicamento, $33,9 \%$ obtiveram sucesso e apenas $0,9 \%$ não apresentaram sucesso na sedação consciente com Midazolam. Entre os pacientes que faziam uso de medicamentos e obtiveram sucesso, $23 \%$ necessitaram de contenção auxiliar para finalizar o tratamento e, entre os que não faziam uso de medicamentos e obtiveram sucesso, $13,5 \%$ necessitaram de contenção auxiliar para finalizar o 
tratamento. Ressaltamos, ainda, que o índice similar de sucesso entre os dois grupos acima citados se deve ao fato de se poder contornar a grande maioria das interações medicamentosas com o midazolam injetável, através de alterações programadas na dosagem da droga sedativa.

Quanto aos efeitos adversos ao uso de midazolam, $\mathrm{EV}$, os principais relatados na literatura disponível são soluços, depressão/parada respiratória, apnéia, redução de tônus muscular (com possibilidade de relaxamento do músculo genioglosso, produzindo obstrução à passagem de ar pela língua - paciente pode engasgar, ou aspirar fluidos) e diminuição de pressão sistólica. 16, 29,30

Muitos desses efeitos puderam ser observados entre os 45 episódios $(9,7 \%$ do total) ocorridos neste estudo, mas, assim como na literatura, em índices muito baixos em relação ao número de procedimentos acompanhados. Ressaltando, mais uma vez, a segurança e eficácia do uso do Midazolam para este fim, assim como a importância de todo o acompanhamento feito ao paciente no CAOE, antes, durante e após o procedimento planejado.

Os efeitos adversos observados foram mínimos. Dentre eles, apenas episódios brandos de queda da saturação de oxigênio, ânsias e/ou vômito, soluços, tosse, presença de secreção pulmonar excessiva, efeito ruim da droga (sem efeito, efeito antagonista ou efeito insuficiente) e taquicardia, corroborando com relatos citados na literatura. Alguns pacientes apresentaram efeitos associados, porém nada digno de nota que tenha atrapalhado a realização dos tratamentos odontológicos planejados.

\section{CONCLUSÃO}

De acordo com os resultados obtidos neste trabalho, podemos concluir que a sedação consciente com midazolam, EV, em pacientes com deficiência pode ser uma boa alternativa nos casos onde as técnicas de manejo de comportamento não são eficazes para a realização de tratamento odontológico, desde que todas a normas de segurança sejam seguidas $e$, que junto a equipe odontológica sempre esteja presente uma enfermeira e o médico responsável pela aplicação do sedativo endovenoso.

\section{REFERÊNCIAS}

1. Girond JBR, Waterkemper R. Sedação, eutanásia e o processo de morrer do paciente com câncer em cuidados paliativos: compreendendo conceito e interrelações. Cogitare Enferm. 2006; 11(3): 258-63.

2. Negro Jr PJ. Sedação consciente e sedação em psiquiatria : paralelos de eficácia e segurança clínicas. Psychiatry on Line Brazil. 2007; 12.

3. Ramos TC, Ramos MESP, Azoubel MCF, Azoubel E, Soares AR, Alves ETP et al. Midazolam: aspectos clínicos e farmacológicos em sedação consciente e estudo retrospectivo avaliando 258 casos clínicos. Innov Implant J. 2009; 4(1):39-45.

4. Yagiela JA, Neidle EA, Dowd FJ. Farmacologia e terapêutica para dentistas. 4. ed. Rio de Janeiro: Guanabara Koogan; 1998. 235-51.

5. Chaia A, Mandarino SCA, Gandelmann IHA, Cavalcante MAA, Pinto LAPF. Valores máximos e mínimos de pressão arterial, frequiência cardíaca e saturação de oxigênio durante as exodontias de terceiros molares inclusos sob anestesia local e sedação prévia. Rev Bras Implant. 2003; 9(2):12-4.

6. Averley PA, Girdler NM, Bond S, Steen N, Steele J. A randomised controlled trial of paediatric conscious sedation for dental treatment using intravenous Midazolam combined with inhaled nitrous oxide or nitrous oxide/sevoflurane. Anaesthesia. 2004; 59(9):844-52.

7. Hulland S, Sigal MJ. Hospital-based dental care for persons with disabilities: a study of patient selection criteria. Spec Care Dent. 2000; 20(4):131-8.

8. Amarante EC, Amarante ES, Guedes Pinto AC. Atualiza-se sobre o uso da sedação consciente por óxido nitroso e oxigênio em odontologia. Rev bras odontol. 2003; 60(2):95-8.

9. Krauss B. Continuous-flow nitrous oxide: searching for the ideal procedural anxiolytic for toddlers. Ann Emerg Med. 2001; 37(1):61-2.

10. Gall O, Annequim D, Benoit G, Glabeke E, Vrancca F, Murat I. Adverse events of premixed nitrous oxide and oxygen for procedural sedation in children. Lancet. 2001; 358(9292):1514-15.

11. Napoli KL, Ingall CG, Martin GR. Safety and efficacy of chloral hydrate sedation in children undergoing echocardiography. J Pediatr. 1996; 129(2):287-91.

12. Vade A, Sukhani R, Dolenga M, Habisohn-Schuck C. Chloral hydrate sedation of children undergoing CT and MR imaging: safety as judged by American Academy of Pediatrics guidelines. AJR Am J Roentgenol. 1995; 165(4):905-9.

13. Parker RI, Mahan RA, Giugliano D, Parker MM. Efficacy and safety of intravenous Midazolam and ketamine as sedation for therapeutic and diagnostic procedures in children. Pediatrics. 1997; 99(3):427-31.

14. Pérez Y, Pardo A, Sequera R, Crespo JD. Sedación y analgesia en pacientes pediátricos para procedimientos fuera del área quirúrgica: artículo de revision. Rev Venez Anestesiol 2001; 6:86-100.

15. Milnes AR, Maupomé G, Cannon J. Intravenous sedation in pediatric dentistry using midazolam, nalbuphine and droperidol. Pediatr Dent. 2000; 22(2):113-9.

16. Agrawal D, Manzi SF, Gupta R, Krauss. Preprocedural fasting state and adverse events in children undergoing procedural sedation and analgesia in a pediatric emergency department. Ann Emerg Med. 2003; 42(5):636-46.

17. Runes J, Ström C. Midazolam intravenous conscious sedation in oral surgery. A retrospective study of 372 cases. Swed Dent J. 1996; 20(1-2):29-33.

18. Richards A, Griffiths M, Scully C. Wide variation in patient response to midazolam sedation for outpatient oral surgery. Oral Surg Oral Med Oral Pathol. 1993; 76(4):408-11.

19. Miyawaki T, Kohjitani A, Maeda S, Egusa M, Mori T, Higuchi $\mathrm{H}$ et al. Intravenous sedation for dental patients with intellectual disability. J Intellect Disabil Res. 2004; 48(Pt 8):764-8.

20. Wilson S. Farrell K, Griffen A, Coury D. Conscious sedation experiences in graduate pediatric dentistry programs. Pediatr Dent. 2001; 23(4):307-14.

21. Wilson KE, Girdler NM, Welbury RR. Randomized, controlled, cross-over clinical trial comparing intravenous midazolam sedation with nitrous oxide sedation in children undergoing dental extractions. Br J Anaesth. 2003; 91(6):850-6.

22. Reyes AM, Alamo RCG. Farmacos utilizados en el stress, ansiedad e insomnio. Málaga: Cooperativa 
Farmacéutica Andaluza; 1997. Disponível em: http://www.cofaran.es/prog9596/temas5_4.html.

Acesso em: 17 de outubro de 2017.

23. Muller S, Prolla JC, Maguilnik I, Breyer HP. Predictive factors of oxygen desaturation of patients submitted to endoscopic retrograde cholangiopancreatography under conscious sedation. Arq Gastroenterol. 2004: 41(3):162-6

24. Allison PJ, Hennequim M, Faulks D. Dental care access among individuals with Down syndrome in France. Spec Care Dent. 2008; 20(1):28-34.

25. American Society of Anesthesiologists Task Force on Sedation and Analgesia by Non-Anesthesiologists. Practice guidelines for sedation and analgesia by nonanesthesiologists. Anesthesiology 2002; 96(4):1004-17.

26. Langlois S, Kreeft JH, Chouinard G, Ross-Chouinard A, East S, Ogilvie RI. Midazolam: kinetics and effects on memory, sensorium, and haemodynamics. Br J Clin Pharmacol 1987; 23(3):273-8.

27. Nishiyama T, Hanaoka K. Accidental overdose of midazolam as intramuscular premedication. J Clin Anesth 2002; 14(7):543-5.

28. Roche Brasil. Monografia do DormonidR (Midazolam). São Paulo: Roche Brasil; 1999. Disponível em: http://www.roche.com.br/ monos/dormonid.doc. Acesso em: 17 out 2017.

29. Barbieri CM, Gomes AC, Menezes TEC, Aguiar SMHCA. Intravenous procedural sedation: an alternative in the treatment of patients with intellectual disability. Braz J Oral Sci. 2010; 9(1):7-10.

30. Collado V, Faulks D, Nicolas E, Hennequin M. Conscious sedation procedures using intravenous midazolam for dental care in patients with different cognitive profiles: a prospective study of effectiveness and safety. PLoS ONE. 2013; 8(8): e71240.

\section{CONFLITO DE INTERESSES}

Os autores declaram não haver conflitos de interesse.

\section{AUTOR PARA CORRESPONDÊNCIA}

Sandra Maria Herondina Coelho Ávila de Aguiar

saguiar@foa.unesp.br

Submetido em $07 / 12 / 2017$

Aceito em 09/01/2018 